\title{
La emocionalidad como elemento de análisis de los nuevos movimientos sociales
}

\author{
Emotionality as an element of analysis of the new \\ social movements
}

\author{
A emocionalidade como elemento de análise dos \\ novos movimentos sociais
}

Recebido em 17-08-2020

Modificado em 08-01-2021

Aceito para publicação 28-03-2021

\section{doi}

https://doi.org/10.47456/simbitica.v8i4.37352

\section{Juan José Labora González}

Doutor em Sociologia pela Universidade de Santiago de Compostela, Espanha. Professor na Universidade de Santiago de Compostela, no Departamento de Ciência Política e Sociologia. Professor colaborador da Universitat Oberta de Catalunya, Espanha, no mestrado de Trabalho Social Sanitário. E-mail: juan.labora@usc.es

\section{Enrique Fernández Vilas}

Licenciado em Ciência Política e Administraçao pela Universidade de Santiago de Compostela, Espanha. Complementa a sua formaçao académica na Universidad de Granada (Espanha), através da Licenciatura em Antroplogia Social e Cultural e na Universidad Nacional de Educación a Distancia com a Licenciatura em Sociologia. E-mail: enriquefernandezvilas@gmail.com

\section{Resumen}

En el presente artículo se analizan las principales emociones y componentes emocionales que tienen influencia en el comportamiento social, a nivel individual y colectivo, y cómo afectan el estudio de los Nuevos Movimientos Sociales (NMS). Frente al racionalismo predominante en el análisis de estos tipos de acción social, se preferencia el análisis del papel de las emociones. Para realizar tales objetivos se lleva a cabo una revisión bibliográfica centrada en el debate entre el racionalismo predominante en el análisis de los movimientos sociales tradicionales y los abordajes que sitúan en el centro de las investigaciones el papel de las emociones en los nuevos movimientos sociales. Por fin, se caracterizan los nuevos movimientos sociales como formas de acción, con algunas características que los diferencian de los movimientos tradicionales, en especial, el hecho de que son movimientos identitarios.

Palabras clave: emociones; nuevos movimientos sociales; acción colectiva; identidad social. 


\section{Introducción}

Si homo es a la vez sapiens y demens, afectivo, lúdico, imaginario, poético, prosaico, si es un animal histérico, poseído por sus sueños y sin embargo capaz de objetividad, de cálculo, de racionalidad, es que es homo complexus.

(Morin, 2014:158)

Aunque siempre han existido movimientos sociales, algún autor ha señalado que "sólo en los siglos XIX y XX los movimientos sociales se han vuelto tan numerosos, tan masivos, $\tan$ sobresalientes y con tantas consecuencias para el curso del cambio" (Sztompka 1995:308). Algo que parece haberse acelerado en los primeros años del siglo XXI que vivimos en la actualidad. A esto se añade el hecho de que el análisis de las emociones a lo largo de la historia, como se verá en el decurso de este artículo, ha estado dominado por un paradigma racionalista que desvirtuaba su estudio. Además, los Nuevos Movimientos Sociales (NMS, de aquí en adelante) imponen la necesidad de ejercer una revisión del papel que pueden jugar en ellos las emociones.

Se han revisado las diferentes definiciones y perspectivas que desde distintas disciplinas se llevaron a cabo para el estudio de los movimientos sociales a lo largo de la historia, mostrando la importancia de los aspectos emocionales. Se realizará una descripción y explicación de las principales emociones y componentes emocionales que tienen influencia en el comportamiento social, a nivel individual y colectivo, y cómo afectan al estudio de los movimientos sociales contemporáneos o NMS teniendo en cuenta que

(...) las emociones están presentes en todas las fases y aspectos de la protesta; motivan a los individuos, se generan en la multitud, se expresan retóricamente y dan forma a los objetivos manifiestos y latentes de los movimientos. Las emociones pueden ser medios, también fines, y otras veces fusionan ambos; pueden favorecer o dificultar los esfuerzos de movilización, las estrategias y el éxito de los movimientos. La cooperación y la acción colectiva siempre han ofrecido la oportunidad de pensar la acción social de una forma más integral; el retorno de las emociones es la última fuente de inspiración para ello (Jasper, 2012a:49).

Así, en este trabajo se lleva a cabo una revisión bibliográfica sobre el racionalismo predominante, y excluyente, en los enfoques y disciplinas que estudiaron el papel de las emociones en los movimientos sociales y cómo estas han asumido un nuevo papel en los NMS (el 15M en España, el MeToo en todo el mundo, la reacción al fallecimiento de George Floyd a escala global, el feminismo, el ecologismo, el movimiento de defensa de los derechos de las personas LGTBI, entre otros). Esta tarea se lleva a cabo desde la aplicación de una estrategia sincrónica y diacrónica a la hora de realizar el análisis. Incidiendo en la consideración, o exclusión, de las emociones como factor explicativo de los movimientos sociales, así como en la valoración positiva o negativa de su posible influencia. 


\section{Movimientos sociales, cambio social y procesos de acción colectiva}

A partir de los años 60 del siglo XX, fueron muchos los movimientos sociales emergentes, sobre todo en Estados Unidos, provocando que el interés académico por esta cuestión creciese hasta que, en la década de los 80 , ya era uno de los principales objetos de estudio de la ciencia política, la sociología y la psicología social.

Consideramos los movimientos sociales como un conjunto de redes de interacción entre diversos actores, informales y basadas en la solidaridad junto con un sentimiento de identificación colectiva. Para ser más precisos, Mario Diani define un movimiento social como "una red de interacciones informales entre una pluralidad de individuos, grupos y/u organizaciones" (1992:8), aprovechando lo que los autores como Eisinger (1973) o Tarrow (2012) denominaron estructura de oportunidad política, una "ventana de oportunidad" que surge y que es condición sine qua non para la organización y potencial movilización de los recursos.

Por otro lado, desde un punto de vista sociológico, los movimientos sociales se han caracterizado como: formas de reconstituir la sociedad (Blumer, 1951), actores históricos (Touraine, 1995), agentes transformadores de la vida política o portadores de proyectos políticos (Eyerman; Jamison, 1991); llegando a considerarse "los movimientos sociales de masas y el conflicto que generan como los agentes primarios del cambio social” (Adamson; Borgos, 1984:12). Es decir, el concepto básico de cambio social se liga al de movimiento social.

McAdam et al. (1999) señalan que un movimiento social tiene la necesidad implícita de superar ciertos objetivos previos al impacto final que pueda tener como sujeto de cambio social:

- Añadir nuevos miembros.

- Mantener la moral y motivación de los ya presentes.

- Ser mediáticos.

- Movilizar a otros grupos.

- Limitar al adversario.

- Influir sobre la política para la actuación del estado.

Asimismo, cabe destacar, que a pesar de que no existe una regularidad ni una forma uniforme de difusión, sí hay una serie de elementos de ciclicidad en los movimientos sociales que Tarrow (2002:103-106) enumera de la siguiente forma:

- Exaltación del conflicto.

- Difusión geográfica y sectorial.

- Desencadenante imprevisible.

- Nuevos marcos de significado y expansión de los repertorios de contestación. 
A lo largo de la historia, fueron muchas las reivindicaciones ciudadanas que tuvieron lugar en diferentes regímenes, ya fuesen estos democráticos o no, en sociedades industriales o preindustriales, pero todos mantenían rasgos comunes de indignación y de necesidad de justicia ante percepciones comunes de ausencia de ella. Así, la génesis de los movimientos sociales se sitúa en la coexistencia de sistemas de valores contrapuestos y de grupos en conflicto mutuo. Los cambios en la estructura social y el orden normativo se interpretan como desviaciones de un proceso de evolución cultural a través del cual nuevas ideas afloran en la mente de los individuos (Della Porta; Diani, 2011).

La participación deja, de esta forma, de ser algo propio de las elites políticas y pasa a ser “cosa de todos/as" y, con el paso del tiempo y la normalización de estas formas de participación, deja de ser un "enemigo del Estado", en un sentido tan negativo o despectivo, y pasa a ser parte fundamental de la lógica democrática, con la aparición de la política de masas (Mazzoleni, 2010).

En el estudio de la acción colectiva, se han distinguido cuatro elementos fundamentales (Funes; Monferrer, 2003), que afectan a los movimientos sociales: intereses, organización, movilización y estructura de oportunidad política. A partir de esto, es preciso establecer el análisis según su nivel de complejidad, para lo que establecemos los siguientes niveles de estudio (Véase tabla 1): micro [sujeto individual], meso [grupo] y macro [sistémico].

Tabla 1 - Formulaciones teóricas para el análisis de la acción colectiva

\begin{tabular}{|c|c|c|c|}
\hline Análisis & Elementos de la acción & Dimensiones del estudio & Formulaciones teóricas \\
\hline Micro & INTERESES & $\begin{array}{l}\text { Características personales. } \\
\text { Aprendizaje de habilidades. } \\
\text { Procesos de socialización. }\end{array}$ & $\begin{array}{c}\text { Elección racional. } \\
\text { Identidad. } \\
\text { Interaccionismo. Etnometodología } \\
\text { simbólica. } \\
\text { Construccionismo. } \\
\end{array}$ \\
\hline Meso & $\begin{array}{l}\text { ORGANIZACIÓN } \\
\text { MOVILIZACIÓN }\end{array}$ & $\begin{array}{l}\text { Estructura organizativa. } \\
\text { Pautas de reclutamiento. } \\
\text { Estrategias. } \\
\text { Repertorios de acción. }\end{array}$ & $\begin{array}{l}\text { Teoría Maximización Recursos } \\
\text { (McAdam, 1982). } \\
\text { Redes Sociales. } \\
\text { Construcción social de la protesta. } \\
\text { Análisis de los eventos de la protesta. }\end{array}$ \\
\hline Macro & $\begin{array}{c}\text { ESTRUCTURA } \\
\text { DE OPORTUNIDAD } \\
\text { POLÍTICA } \\
\\
\text { ESTRUCTURA } \\
\text { DEL CONTEXTO }\end{array}$ & $\begin{array}{l}\text { Sistema político. } \\
\text { Sistema social. } \\
\text { Sistema económico. } \\
\text { Sistema cultural. }\end{array}$ & $\begin{array}{c}\text { Teorías de la democracia. } \\
\text { Capital social. } \\
\text { Proceso político. } \\
\text { RRSS. } \\
\text { NMS. } \\
\text { Sociedad del riesgo (Beck, 2013). } \\
\text { Cambio social. }\end{array}$ \\
\hline
\end{tabular}


Estas dimensiones permiten a su vez distintas clasificaciones dentro de los movimientos sociales según el criterio diferenciador que se use. Así, por ejemplo, Sztompka (1995) establece una clasificación en función de las formas de organización, sus objetivos y alcance:

- Movimientos reformistas. Pretenden un relativo cambio en la sociedad sin modificar básicamente su estructura institucional.

- Movimientos radicales. Sus objetivos son los de provocar un cambio absoluto en aquellos aspectos de la organización social con los que no están de acuerdo.

- Movimientos revolucionarios. Los cambios deseados afectan a la estructura social en sí misma.

Por otro lado, usando un criterio histórico, hablaríamos de (Monferrer, 2006):

- "Viejos" movimientos sociales: movilizados por intereses de clase.

- "Nuevos” movimientos sociales, cuya aparición viene acompañada de demandas posmaterialistas e interclasistas.

- "Novísimos" movimientos sociales: movilización para la consecución de un modelo alternativo de justicia global.

Finalmente, si se usa como criterio clasificador la cualidad del cambio, podemos distinguirlos entre movimientos progresistas y movimientos conservadores (Diz et al., 2004). En la citada tipología podemos encuadrar tanto a los movimientos sociales que tanta relevancia tuvieron en décadas anteriores como a los NMS. En su estudio, prevaleció durante años la perspectiva materialista proveniente de la teoría marxista y funcionalista (de la Garza, 2011), la cual insistía en una tesis basada en una movilización que dependía de los recursos. Las luchas en las sociedades industriales eran, en su mayoría, de corte obrerista, buscando mejores condiciones laborales. La participación en ellas era inherentemente materialista, sin tener en cuenta variables explicativas de carácter psicosocial. Este enfoque psicosocial aparece en la sociología funcionalista (Ullán, 2016). Las teorías basadas en la elección racional del individuo, como era la ya mencionada estructura de oportunidad política (Eisinger, 1973; Tarrow, 2012), la idea del free-rider de Olson (1965) o las teorías de la movilización de recursos (Mcadam, 1982; Oberschall, 1995; Perrow y Jenkins, 1977; Tilly, 1978) basadas en dos enfoques diferenciados: el empresarial (Mccarthy; Zald, 1977) y el político (Tilly, 1978), son superadas para dar paso a nuevas formas de organización y nuevos objetivos de acción.

\section{La aparición de los Nuevos Movimientos Sociales}

Pese a una dinámica continuada centrada en los estudios de la Rational Choice, -cuya naturaleza reside en el análisis económico- en los años noventa, algunos investigadores/as de la ciencia política colocaron de nuevo el foco en el papel desempeñado por las emociones en la 
producción y reproducción de movimientos sociales centrado en los NMS. A su entender, la producción simbólica no está orientada sólo estratégicamente, sino que implica sentimientos y emociones. El choque moral motivado por la quiebra de reglas y normas profundamente arraigadas es a menudo el primer paso para la movilización individual y, de hecho, las organizaciones de protesta se dedican a transformar el miedo en rabia e indignación moral (Jasper, 1997). Atrás quedan las principales teorías por las que se movían los movimientos sociales "tradicionales" que, aunque se nutrían de cierta influencia neomarxista, estaban centradas en los movimientos sociales urbanos y su búsqueda del bienestar y el acceso a servicios en los estados occidentales (Castells, 1977a, 1977b).

En estos nuevos y novísimos movimientos sociales que han ido surgiendo se han distinguido nuevas características que los diferencian de algunos de los movimientos anteriores. De acuerdo con Sztompka (1995):

- Se ocupan de problemas nuevos: surgen nuevos intereses y nuevas fuentes de conflicto social. Además de las tradicionales reivindicaciones de tipo económico aparecen algunas nuevas relacionadas con la identidad de grupo, derechos de la sociedad civil, entre otras; en definitiva, valores de carácter no económico o posmaterialistas.

- Las personas participantes no se pueden identificar directamente con una clase social: los intereses que generan el conflicto o la necesidad de cambio son comunes a varias clases o grupos sociales. Kriesi (1989), por ejemplo, señala que es la sobrerrepresentación de personas con un nivel educativo alto y de las clases medias, argumentándose que este sector de población estaría más sensibilizado y dispondría de más tiempo a disposición para acudir a una movilización o implicarse en las reivindicaciones sociales.

- Los NMS suelen ser descentralizados: en lugar de la típica organización vertical y jerárquica de los movimientos sociales tradicionales, surgen movimientos organizados de forma reticular, sin centro aparente, y sin la rigidez que caracterizaba a los movimientos históricos.

A esto se añade el que en los NMS los valores (véase tabla 2) materialistas y posmaterialistas se mezclan (Inglehart, 1971, 1998). Aunque en las sociedades industriales avanzadas, como la que estudiamos en este trabajo, tienden a dar una importancia menor a los valores materialistas, llevando a cabo un cambio cultural (Montero; Torcal, 1995) en pro de los valores de corte no-económico. 
Tabla 2. Paralelismo entre el índice de Inglehart y diferencias entre nuevos y viejos movimientos sociales

\begin{tabular}{|c|c|}
\hline $\begin{array}{c}\text { Valores materialistas de los viejos } \\
\text { movimientos sociales }\end{array}$ & $\begin{array}{c}\text { Valores posmaterialistas de los nuevos } \\
\text { movimientos sociales }\end{array}$ \\
\hline Mantenimiento del orden & Participación ciudadana en cuestiones que les conciernen \\
\hline Luchas contra la inflación & Participación ciudadana en decisiones políticas \\
\hline Estabilidad económica & Protección de la libertad de expresión \\
\hline Lucha contra la delincuencia & Protección medioambiental y urbana \\
\hline Crecimiento económico & Humanización de la sociedad \\
\hline Fuerzas armadas poderosas & Progreso humanístico \\
\hline
\end{tabular}

Fuente: adaptado de Ullán (2016).

En este sentido, Boaventura de Sousa considera que

(...) la novedad más grande de los NMS reside en que constituyen tanto una crítica de la regulación social capitalista, como una crítica de la emancipación social socialista tal como fue definida por el marxismo. Al identificar nuevas formas de opresión que exceden las relaciones de producción, y ni siquiera son específicas de ellas, como son la guerra, la polución, el machismo, el racismo o el productivismo; y al abogar por un nuevo paradigma social, menos basado en la riqueza y en el bienestar material del que, en la cultura y en la calidad de vida, denuncian los NMS (De Sousa, 2001:178).

Melucci (1980 y 1982) entendió, como se ha apuntado en las líneas precedentes, que los NMS pretenden crear mayor autonomía personal y no limitarse a fines de tipo material, conformando construcciones en la medida que la acción social es creada y activada. Este constructivismo tiene su máxima expresión en Touraine $(1994,1995)$ basado en la idea de que en las sociedades posindustriales, los movimientos sociales pueden modificar la sociedad mediante la acción, no atacando directamente al sistema político, sino construyendo identidades. Así, Melucci (1989) describe la acción colectiva como producto de un sistema formado por 1) las metas de la acción colectiva, 2) los medios de acción, 3) el espacio de la acción.

Así, Melucci (1996) construye un modelo analítico en las sociedades complejas, basado en que a) los individuos deben ser capaces de manejar gran cantidad de códigos y reglas, dada la cantidad de ámbitos en los que se mueven, b) las sociedades mudan rápidamente y c) las oportunidades de acción son abiertas, la aceleración del cambio excede la capacidad de acción de los actores movilizados.

Por otro lado, este mismo autor (Melucci, 2001) expone que sí existe un objetivo común, pero que si algo caracteriza a los NMS es la negativa al manejo de la vida individual por parte de factores externos, ergo, reivindican la autonomía de esos individuos. Con todo, Melucci (1995) 
veía la acción como una orientación con propósito definido, la cual se prepara dentro de un sistema de oportunidades y limitaciones.

Claus Offe (1992) expone que los principales problemas de los NMS son:

- El apartamiento de la clase obrera industrial y de los sectores más vinculados a la crítica intelectual es una consecuencia de la falta de una interpretación de la realidad que establezca las condiciones de la transformación y superación de la misma.

- Los actores que conforman estos movimientos: entendiendo que el interclasismo de muchos de ellos, aparta al propio movimiento de un interés y objetivo común.

Nos centraremos a partir de este punto, en los NMS, más concretamente en los de protesta, dado que estos encarnan especialmente las características de las que se dotan los movimientos sociales en el siglo XXI con la Sociedad Red (Castells, 1996, 2006).

Entendemos la protesta como los movimientos llevados a cabo por aquellos sectores de la sociedad civil que carecen de poder institucional, en tanto que "las desigualdades, el autoritarismo, la corrupción y el funcionamiento de los servicios públicos han concitado una gran energía contra el orden establecido” (Billion; Ventura, 2020:37). Para ser más precisos, Taylor y Van Dyke (2004:268) definen las protestas como "espacios de contestación donde se utilizan cuerpos, símbolos, identidad, prácticas y discursos para perseguir o prevenir cambios en las relaciones institucionalizadas de poder". Se trata también, de movimientos que utilizan canales indirectos para ser influyentes en el proceso de toma de decisiones y de producción de políticas públicas.

De esta forma, los movimientos de protesta son los más habituales dentro de los movimientos sociales, albergando multitud de formas y mecanismos de actuación, mudados y modernizados con la aparición de la web 2.0. Las conexiones entre personas y organizaciones fueron objeto de un cambio radical por los cambios que se han producido en la comunicación.

Es el auge de lo que Castells (2012) denominó autocomunicación de masas, basada en redes horizontales de comunicación interactiva y multidireccional en internet y, cada vez más, en redes de comunicación inalámbricas, la plataforma de comunicación prevalente en la actualidad a escala global. Las antiguas redes centralizadas se convirtieron en redes distribuidas y, por tanto, la comunicación ya no es unidireccional, en lo que entendemos por Sociedad Red.

El propio Castells (2012) expone una serie de características que comparten los movimientos derivados de las nuevas formas de comunicación:

- Son espontáneos en su origen, por tanto, no existe una génesis clara y premeditada, sino que son motivo de una acción concreta en un momento determinado. 
- Son virales, es decir, se da una difusión imposible de lograr en cualquier otro contexto histórico, lo que también contribuye a su transnacionalización.

- Afán de ser movimientos sin un líder claro. Cualquier delegación de poder indigna a sus participantes, ya que se reflejarían las relaciones de poder que se dan con las élites políticas.

- Las redes horizontales multimodales dan lugar a la unidad. Se descubren muchos valores en común gracias a la acción en comunidad.

- Son movimientos altamente reflexivos y no violentos.

- No son movimientos programáticos, ya que engloban una gran cantidad de causas separadas e interrelacionadas y el grueso de sus reivindicaciones tiene que ver con la mejora de su calidad de vida.

- Por último, como tienen lugar en una red de redes, se trata de movimientos sin un centro identificable, que se desarrollan de forma física en espacios urbanos a posteriori. El híbrido resultante del ciberespacio y el espacio urbano ocupado constituye un tercer espacio, el espacio de autonomía.

Estos movimientos pasan a ser locales y globales de forma simultánea, se convierten en transnacionales no sólo por su temática, sino debido a la rápida transmisión por redes. YouTube o Facebook fueron clave en los movimientos dados en la última década en Túnez, Egipto, Nueva York o Madrid, haciendo públicas imágenes que provocaban aún mayor indignación en la opinión pública y ponían sobre la mesa problemas que traspasaban el ámbito nacional. La dimensión planetaria que alcanzan los movimientos sociales del siglo XXI es, por tanto, la principal característica que los diferencia de las antiguas formas de movilización. Está claro que la dimensión global de estos movimientos no es algo totalmente nuevo, pero sí de un amplio debate (Alonso, 2009). El movimiento estudiantil de los sesenta, el movimiento feminista de los setenta y los movimientos pacifistas y ecologistas de los ochenta son ejemplos de los que se llamaron movimientos "globales". Si retrocedemos aún más, "las revoluciones de 1848 y el movimiento antiesclavista fueron fenómenos colectivos que en su desarrollo traspasaron los límites continentales" (Della Porta; Diani, 2011:139). Aun con todo, esta internacionalización de los movimientos no es comparable con la capacidad que cualquier estructura orgánica que se movilice en la actualidad tiene para exportar el discurso a otros lugares y a velocidades impensables pocas décadas atrás.

Por contra, lo que une a viejos y nuevos movimientos sociales es que un movimiento de acción colectiva siempre busca un cambio, es decir, un cambio (dentro) del sistema en el que se encuadra o incluso un cambio de sistema. En el grueso de los casos, son modificaciones 
sectoriales, ergo, con beneficiarios limitados que llegan a verse recompensados por la participación en los movimientos con cambios en las políticas públicas.

\section{La emoción como variable de análisis}

Las emociones son un asunto que aparece en los albores del pensamiento occidental. De hecho, a lo largo de la historia de la filosofía occidental, fueron muchos los/as autores/as que, de una u otra forma, pusieron el foco de sus obras en torno de esta cuestión. En su estudio, se pueden distinguir las siguientes posturas: la búsqueda de la armonía emocional, la reivindicación de determinadas emociones consideradas "positivas", es decir, aquellas que provocan alegría o placer y, señalando, en cualquier caso que sea las emociones deben experimentarse con moderación (Casado; Colomo, 2006). Finalmente Ferrater (1988), en su influyente diccionario, señala que ha sido muy habitual el que se intentase negar o descartar los aspectos emocionales.

El conocimiento, si lo decimos en lenguaje típicamente cartesiano, sólo proviene de las ideas claras y distintas (Descartes, 1989), esto tuvo a las emociones apartadas, y separadas, de la teoría del conocimiento durante siglos.

En disciplinas con mayor tradición que en las ciencias sociales, como son las 225 disciplinas/saberes psi -utilizando la denominación de Castel $^{1}$ (Castel; Castel; Lovell, 1982)- el interés por el afecto y la emoción ha sido muy desigual a lo largo del tiempo. En un texto escrito en 1996, se puede leer:

\footnotetext{
Durante los dos primeros tercios de este siglo [refiriéndose al siglo XX] la psicología se desentendió de la vida afectiva, mientras que la psiquiatría y la neurología conservaron el interés. En los años treinta algunos psicólogos -por ejemplo Duffy y Meyerpredijeron que el término desaparecería de la psicología, predicción que estuvo a punto de cumplirse en los setenta (Marina, 2005:23-24).
}

Un poco más adelante este mismo autor matiza que con el tiempo esta situación cambió, calificando de "vivo e intenso" (Marina, 2005:25) el interés por el mundo de la afectividad. Marina (2005) cita como ejemplos de esta situación a: Arnold, Lazarus, Tomkins, Ekman, Izard, Mandler, Fridja, Ortony, Oatley, Johnson-Laird, Zajonc, Pribram, Davidson, Le-Doux, Leventhal, Lang, Scherer, Averill, Harré y Harris. Las investigaciones sobre las emociones serían de cuatro tipos: “evolutivo, psicofísico, dinámico y constructivista” (Marina, 2005: 25).

En 2012, Ledoux explica la evolución del interés por las emociones desde la neurociencia y, curiosamente, la situación es muy parecida, en el sentido del interés creciente por las emociones, a la comentada por Marina. Ledoux (2012:35) señala que "hace veinte años nadie

\footnotetext{
${ }^{1}$ Robert Castel acuñó este término para referirse a los/las profesionales de las disciplinas relacionadas con la psique humana: psicología, psiquiatría, psicoterapia, trabajo social, etc.
} 
prestaba atención a las emociones y el cerebro, pero parece que, en los últimos dos años, ha habido una auténtica oleada de actividad al respecto". En cualquier caso, en el campo de las disciplinas psi, a pesar de admitirse cada vez más la influencia de los aspectos sociales y culturales, las emociones son consideradas en su significado esencialmente biológico (Damasio, 2013; Maturana, 1997), es decir, se ajustarían al imaginario biologicista del modelo médico (Labora, 2018; Torres, 2012; Tyreer; Steinberg, 1987).

Desde las disciplinas psi se coincide en caracterizar las emociones como elementos activos, no pasivos en el conocimiento (Humphrey, 2012). Una segunda característica destacada es que no se pueden separar de la cognición (Ledoux, 2012). Curiosamente eso coincidiría con lo apuntado desde hace tiempo por parte de distintos filósofos (Gabriel, 2019; Zubiri, 1982, 1983, 1991). De esta manera se vuelve a unir lo que desde Descartes, como se ha comentado, se había intentado separar.

Esta unión permite, por otro lado, alejar el fantasma que sobrevolaba desde el inicio de la psiquiatría, es decir, el considerar las emociones como la causa de la enfermedad mental. Ya Pinel y los llamados alienistas mantenían esta idea (Scull, 1989). Esto introdujo la idea de que las mujeres eran más propensas a padecer algún tipo de enfermedad mental (Appignanesi, 2009; Ussher, 1991). Y, además, hizo que las emociones y los sentimientos se impregnasen de un aura de mala prensa que incluso se mantiene hoy en día en algunos/as autores/as que siguen hablando del ser humano como un ente que debería de ser totalmente racional y cuyos errores y sesgos provienen de su parte afectiva.

También las ciencias sociales dedicaron sus esfuerzos al estudio de las emociones. Estas han sido consideradas predominantemente como motivadoras de acción (Elster, 2010). Así, la sociología de las emociones es una subdisciplina que se viene trabajando desde hace tiempo (véase tabla 3). Una de las novedades que aportan las ciencias sociales al estudio de las emociones es el considerarlas no solamente como un fenómeno individual, sino que estas se enfocan como fenómenos colectivos, que pueden, incluso, generar fenómenos de contagio; y crear, o modificar, climas emocionales que pueden ser positivos o negativos (Bisquerra, 2017). Además, se incluye de lleno la identidad como algo a tener en cuenta en el estudio de las emociones y de los movimientos sociales. La identidad se ve influida y constituida por las elecciones que tomamos y el compromiso personal que podamos asumir en un momento dado con determinadas causas (Castells, 2013; Guibernau, 2017). 
Tabla 3 - Las emociones en la sociología

\begin{tabular}{|l|l|}
\hline $\begin{array}{l}\text { Cuthberth-Jhonson, Beverly, Franks, David D. y } \\
\text { Dornan, Michael (1994) }\end{array}$ & $\begin{array}{l}\text { Sociology of Emotions: An Annotated Bibliography. } \\
\text { Nueva York: Garland Publishing. }\end{array}$ \\
\hline Berry, Bonnie (1999) & $\begin{array}{l}\text { Social Range: Emotion and Cultural Conflict. } \\
\text { Abingdon: Routledge. }\end{array}$ \\
\hline Barbalet, Jack (2002) & $\begin{array}{l}\text { Emotions and Sociology. Nueva Jersey: Willey- } \\
\text { Blackwell. }\end{array}$ \\
\hline Flam, Helena y King, Debra (Eds.) (2005) & $\begin{array}{l}\text { Emotions and Social Movements. Abingdon: } \\
\text { Routledge. }\end{array}$ \\
\hline Tenhouten, Warren D. (2008) & $\begin{array}{l}\text { A General Theory of Emotion and Social Life. } \\
\text { Abingdon: Routledge. }\end{array}$ \\
\hline Turner, Jonathan H. y Stets, Jan E. (2011) & Sociology of Emotions. Cambridge University Press. \\
\hline Von Schere, Christian y Salmella, Mikko (2014) & $\begin{array}{l}\text { Collective Emotions: Perspectives from Psychology, } \\
\text { Philosophy and Sociology. Oxford University Press. }\end{array}$ \\
\hline Harris, Scott R. (2015) & $\begin{array}{l}\text { An Invitation to the Sociology of Emotions. Abingdon: } \\
\text { Routledge. }\end{array}$ \\
\hline Stets, Jan E. y Turner, Jonathan H. (Eds.)(2015) & $\begin{array}{l}\text { Handbook of Sociology of Emotions. Amsterdam: } \\
\text { Springer. }\end{array}$ \\
\hline Franks, David D. (2017) & Sociology of Emotions. Londres: Elsevier. \\
\hline Von Schere, Christian (2017) & $\begin{array}{l}\text { Emotion and Social Structures: The Affective } \\
\text { Foundation of Social Order. Abingdon: Routledge. }\end{array}$ \\
\hline Jacobsen, Michael H. (2019) & $\begin{array}{l}\text { Emotions, Everyday Life and Sociology. Abingdon } \\
\text { Routledge. }\end{array}$ \\
\hline Demertzis, Nicolas (2020) & $\begin{array}{l}\text { The Political Sociology of Emotions. Essays on } \\
\text { Trauma and Resentments. Abingdon: Routledge. }\end{array}$ \\
\hline
\end{tabular}

Fuente: elaboración propia.

Todo esto permite ampliar el foco biomédico e incluir en el estudio de las emociones elementos que las conecten con la acción, y en este caso una acción ya colectiva no sólo individual. Hacen aparición así aspectos tales como: las tendencias a la acción, la consideración de las emociones como algo de carácter intencional, es decir, que se refieren a algo, considerándose además las emociones como generadoras de evaluaciones del entorno a partir de su carácter positivo o negativo (Elster, 2010). Por otro lado, las mencionadas tendencias a la acción de cada emoción serían distintivas de cada una de ellas; estando por esto algunas de ellas muy vinculadas a las normas morales y sociales (Elster, 2010). Además, las emociones pueden generar creencias y también pueden llegar a alterar la jerarquía de motivaciones de una persona ya que, como señala Elster (2010) hay determinadas emociones negativas que pueden afectar a las motivaciones de la persona que las siente.

Sin embargo, cuando hablamos de emociones o de influencia emocional de algo o sobre algo, estamos penetrando en un campo tratado escasamente por la ciencia política. El hiperracionalismo de la disciplina (Máiz, 2010), cuyos teóricos no son capaces de escapar de lo racional, no se exponen a separar las emociones como una variable aparte. Pero, como señala Sutil: 
Desde la ontología relacional (externalista, anticartesiana) se puede afirmar que la emoción -y sus conceptos asociados- nunca es un fenómeno puramente individual sino una comunicación entre dos o más personas y el lugar en el que hay que analizarla, por tanto, es en el contexto de la conducta comunicativa y su significado (2013:349).

Entonces, para poder entender las lógicas que siguen los movimientos sociales y de protesta, es necesario analizar y comprender qué emociones se dan en cada momento y contexto, y qué papel juegan en el transcurso del mismo, escapando del hiperracionalismo que se explicaba en líneas anteriores, comprendiendo las emociones como fenómenos sociales, resultantes de lógicas constructivistas de significación e interpretación.

En cualquier caso, el concepto "emoción" se emplea habitualmente para describir multitud de fenómenos, conexos, pero diferentes como: sentimientos, pasiones, temperamentos, afectos, entre otros. En este trabajo nos hemos referido al campo de las emociones en sentido amplio. En la actualidad la literatura científica coincide en el hecho de considerar las emociones como fuentes de información que nos permiten realizar evaluaciones de la realidad, funcionando como motivadoras de acción a través de las tendencias que originan (Ekman, 2004; Marina, 2005).

En principio, se distinguieron seis emociones básicas: asco, miedo, sorpresa, alegría, ira y tristeza (Ekman; Levenson; Friesen, 1983). A partir de los años 90 el propio Ekman (1999) se corrigió a sí mismo ampliando este número a diez: diversión, desprecio, satisfacción, vergüenza, emoción, culpa, orgullo por el logro, alivio, satisfacción y placer sensorial. Posteriormente se ha planteado la posibilidad de que solamente sean cuatro las emociones básicas, siendo el resto modalizaciones, cronológicamente posteriores, de las primigenias (Jack; Garrod; Schyns, 2014).

Jasper (2012a) nos ofrece una tipología básica más relevante en relación al objeto de este artículo. Este autor expone la existencia de estados de ánimo relativamente estables y de largo recorrido, que a menudo constituyen el trasfondo del estado de ánimo y las emociones reflejas: 1) compromisos o lealtades afectivas: sentimientos relativamente estables, positivos o negativos, en relación con otros o sobre objetos, tales como el amor y el odio, el agrado y el desagrado, la confianza o desconfianza, el respeto o el desprecio; y, 2) emociones morales: sentimientos de aprobación o desaprobación basados en instituciones o principios morales, tales como la vergüenza, la culpa, el orgullo, la indignación, la furia y la compasión.

Las emociones se relacionan en el cerebro con la parte cognitiva, en estructuras relacionadas entre sí. Desde esta perspectiva, basada en la cognición, se equiparan las emociones a la razón. En ese sentido, autores como Lazarus (1993) defienden que no existe una emoción sin razón, y, ante todo, que nuestras emociones son el producto de la interpretación, es decir, del proceso cognitivo que tiene lugar en nuestro cerebro sobre la realidad que vivimos. 
En resumen, se puede decir que existe una serie de emociones básicas que, posteriormente, se combinan entre sí (véase tabla 4). Estas nos proporcionan una valoración axiológica organizada de la realidad, que funciona, a su vez, como elemento movilizador y motivador de cara a una acción determinada. Marina y López (1999) reducen este dinamismo emocional a cuatro tendencias: impulso a ir hacia algo bueno (atracción), impulso a ir contra algo (agresión), impulso a separarse de algo (aversión) e impulso a separar algo de mí (repugnancia).

Tabla 4. Emociones avanzadas como combinación de emociones básicas

\begin{tabular}{|c|c|c|}
\hline Suma emociones básicas & Emoción avanzada & Opuesta avanzada \\
\hline Alegría + anticipación & Optimismo & Decepción \\
\hline Confianza + alegría & Amor & Remordimiento \\
\hline Miedo + confianza & Sumisión & Desprecio \\
\hline Sorpresa + miedo & Susto & Alevosía \\
\hline Tristeza + sorpresa & Decepción & Optimismo \\
\hline Aversión + tristeza & Remordimiento & Amor \\
\hline Ira + aversión & Desprecio & Sumisión \\
\hline Anticipación + ira & Alevosía & Susto \\
\hline
\end{tabular}

Fuente: Plutchik (2001); Maneiro (2017).

En cualquier caso, Plutchik (2003) defiende que debemos considerar las emociones desde una perspectiva más bien amplia. Desde este punto de vista, de carácter holístico, las emociones se pueden definir a partir de la posesión de siete características (Elster, 2002: 299): a) parten de una sensación cualitativa; b) poseen antecedentes cognitivos; c) son intencionales; d) parten de alguna excitación fisiológica; e) provocan expresiones fisiológicas; f) provocan valencias en la dimensión placer-dolor; y g) provocan tendencias de acción características.

Así, una vez tenemos definidas las emociones y los movimientos sociales, la pregunta es si estas son necesarias para el análisis de los procesos de acción colectiva, puesto que nos encontramos inmersos en un marco sociopolítico donde todo apunta a un cambio no solo epistemológico, sino también axiológico: el giro afectivo.

\section{El giro afectivo: inteligencia afectiva y cognición}

De un tiempo a esta parte, la emoción ha pasado a conferir una nueva perspectiva en el análisis de las dinámicas políticas y de las lógicas democráticas y de protesta, frente al hiperracionalismo antes citado. Aparece una mayor "tendencia hacia la subjetivación” (Sánchez, 
2016). A este nuevo paradigma se le ha venido denominando giro afectivo (The Affective Turn).

El giro afectivo es, en primer lugar, la reacción del mundo de la investigación a un cambio más general, es decir, a la emocionalización de la vida pública -y de las instituciones-, sectores y subsistemas que la conforman. Este término se refiere al creciente y crucial papel de las emociones en la transformación de esferas de la vida pública tales como: los medios de comunicación, la salud, o la esfera legal entre otras. Corinne Squire (2001) bautizó a estas sociedades como sociedades afectivas.

Podríamos resumir el giro afectivo como "un cambio en la concepción del afecto que vino a modificar la producción de conocimiento y la lógica misma de las disciplinas" (Enciso; Lara, 2013:102), contrariamente a los estudios con fundamento epistemológico en la teoría de la acción racional (Rational Choice Theory, RCT), en tanto que (Jaspers, 2012b:11-12)

[...] las criticas teóricas apuntaban a que los supuestos de la teoría de la elección racional a menudo estaban insertados subrepticiamente en los modelos (Jasper, 1997; Opp, 2009); a qué conceptos tales como los de recursos y oportunidades políticas estaban ampliamente diseminados (Gamson; Meyer, 1996); a que el concepto de oportunidades combinaba coyunturas estratégicas de corto plazo y horizontes estructurales de largo plazo (Jasper, 2012c); a que el paradigma hacía que las protestas se viesen muy fáciles y normales (Piven; Cloward, 1992); a que los rumbos estructurales no permitían fijar plenamente la atención en las dinámicas culturales (Goodwin; Jasper, 2004); y que las emociones estaban de todo ausentes (Goodwin; Jasper; Polletta, 2000).

Esta supuesta ausencia, se postula problemática para el análisis actual, con la llegada de nuevas formas de acción estudiadas desde una nueva óptica: la afectiva.

Para poder entender la llegada de los estudios sobre inteligencia afectiva en numerosas disciplinas, que marcan una nueva perspectiva a la hora de explicar determinados comportamientos, es imprescindible hacer primero una pequeña revisión histórica. Las aproximaciones del siglo XX a estos estudios son las siguientes (Enciso; Lara, 2014):

- El socioconstruccionismo, que "libera" las emociones de sus raíces biologicistas.

- La psicología social discursiva, la cual pasa a centrarse únicamente en el lenguaje.

- Los estudios culturales de las emociones que encaminaron el giro afectivo hacia los estudios contemporáneos con dos acciones concretas: la propuesta de la variabilidad cultural y la atribución emocional que puede tener un amplio espectro de interpretación en función del contexto.

- Las emocionologías. Concepto creado por Peter Stearns y Carol Z. Stearns (1985), quienes se basaron en la perspectiva histórica para referirse a los modos en que la gente de una cultura particular identifica y reconoce emociones.

- La sociología interpretativa, la cual explicita que las emociones tienen una relación determinante con las estructuras sociales. 
- La sociolingüística de las emociones, mediante la idea de que el pensamiento se estructura en términos de lenguaje (De Saussure, 1987). Así, las emociones existen previas a impactar el lenguaje y no son construidas en éste, sino expresadas a través de él.

- Los estudios feministas de las emociones. El feminismo adapta: la disolución de la díada razón-emoción de Spinoza (2014), la problematización de la asociación emoción a femenino y razón a masculino, y el concepto de la experiencia como fuente productora de conocimiento.

De nuevo hallamos vinculadas las emociones a la acción. En este sentido la teoría de la inteligencia afectiva propone una ruta indirecta para unir emociones, cognición y acción. En ella, las emociones sirven como una señal para procesar información de manera sistemática o confiar en predisposiciones en el momento de definir la dirección de la acción participativa.

De acuerdo con el trabajo de George Marcus (2003), las emociones entran dentro de dos sistemas ortogonales: el sistema de disposición y el sistema de vigilancia. El sistema de disposición se refiere a una constelación de emociones que provee guía directa y facilita orientaciones enfocadas al comportamiento; cuando los individuos se encuentran en este estado, tienden a confiar en hábitos políticos, tales como el voto partidario, debido a que no existe nada en el contexto que los motiva a utilizar pistas alternativas. Por otro lado, cuando se activa el sistema de vigilancia, usualmente mediante la introducción de un estímulo externo, los individuos confían menos en predisposiciones y hábitos, utilizando nueva información para la toma de decisiones (Becerra, 2016).

Algunos autores afirman que "el conocimiento tradicional sostiene que la gente emocionalmente comprometida tiene menos probabilidades de tomar decisiones racionales" (Marcus et al., 2007:254). Con todo, este axioma depende de lo que entendamos como "comprometida emocionalmente"; si queremos decir más ansioso, entonces la teoría de la inteligencia afectiva argumenta que el compromiso emocional motivará a la gente para tomar decisiones políticas más razonadas que quienes permanecen desapasionados, lo que, referido a lo que estamos tratando en este trabajo, supone la contraposición a la creencia de la toma de decisiones mediante el análisis coste-beneficio. Los elementos emocionales ganan peso en las explicaciones sobre el surgimiento en la acción colectiva y la participación política, entendiendo que el compromiso para participar puede ser incluso mayor cuando hay un alto componente emocional.

Afirmaba Paul Ekman (2017:36-37) que ante la posibilidad de una única reacción ante un igual estímulo emocional "está claro que eso no es así [...] no respondemos emocionalmente a todo; no estamos continuamente paralizados por la emoción. Las emociones van y vienen [...] hay personas mucho más emotivas que otras". Y es que las diferentes respuestas a una misma 
emoción provocarán también diferentes desarrollos de la acción. Por una parte, debido a un componente propio, individual, particular de cada individuo y su propia psique, una cuestión que introducíamos en líneas previas. Por otra, por variables más amplias, socioestructurales, y en relación con un determinado esquema cultural. Por ello, el análisis en esta línea cobra todavía mayor importancia a la hora de entender las diferentes subjetividades, el desarrollo de la acción y, finalmente, las dinámicas sociopolíticas que tengan lugar.

\section{Conclusiones}

Después del recorrido efectuado podemos concluir que frente al racionalismo que ha dominado en el análisis de los movimientos sociales, en los últimos tiempos la literatura científica, desde distintos ámbitos, plantea la necesidad de contar con las emociones como elementos motivadores y movilizadores de estos tipos de acción social, especialmente de los que vienen surgiendo en los últimos tiempos.

Por otro lado, se ha podido ver que otro aspecto importante a incorporar en los estudios sobre los NMS es la consideración de estos como formas de acción en los que se incluyen no sólo reivindicaciones, predominantemente, de tipo socioestructural o económico, sino que los NMS se constituyen como movimientos identitarios para cuyo análisis se hace patente la necesidad de incluir los aspectos emocionales de los seres humanos.

La incorporación de estos dos aspectos a la investigación de los NMS se considera que puede ayudar a dotar de mayor complejidad a los análisis de los nuevos movimientos que vienen surgiendo de manera profusa en años recientes y que reclaman estudios complejos de las relaciones y las transacciones sociales establecidas por parte de las personas en esta sociedad hiperconectada y saturada de información en la que nos movemos.

\section{Bibliografía}

ADDAMSON, Madeleine; BORGOS, Seth (1984), This Mighty Dream: Social Protest Movements in the United States. Boston, Routledge.

ALCÁNTARA, Manuel (2014), "Neuropolítica: una aproximación a la micropolítica". Revista Española de Ciencia Política. Madrid, v. 35, pp. 31-55 [Consult. 28-02-2021]. Disponible en https://recyt.fecyt.es/index.php/recp/article/view/37625/21143

ALONSO, Angela (2009), "As teorias dos movimientos sociais: um balanço do debate". Lua Nova, 76, pp. 49-86 [Consult. 10-12-2020]. Disponible en https://www.scielo.br/pdf/ln/n76/n76a03.pdf

APPIGNANESI, Lisa (2009), Mad, Bad, Sad. A History of Women and the Mind Doctors from 1800 to the Present. Londres, Virago. 
BECERRA, María G. (2016), "El voto emocional. Un análisis del rol de las emociones en el comportamiento político del elector peruano". Cuadernos de investigación. Lima, v. 13, pp. 1-55 [Consult. 10-09-2020].

Disponible en http://repositorio.pucp.edu.pe/index/bitstream/handle/123456789/54905/Cuadernos\%20de\%20investig aci\%C3\%B3n\%2013.pdf?isAllowed=y\&sequence $=1$

BECK, Ulrich (2013), La sociedad del riesgo. Hacia una nueva modernidad. Barcelona, Paidós.

BILLION, Didier; VENTURA, Christophe (2020), ¿Por qué protesta tanta gente a la vez? Nueva Sociedad. Buenos Aires, 286, pp. 37-52 [Consult. 15-06-2020]. Disponible en https://nuso.org/articulo/por-que-protesta-tanta-gente-la-vez/

BISQUERRA, Rafael (2017), Política y emoción. Aplicaciones de las emociones a la política. Madrid, Pirámide.

BLUMER, Herbert (1951), "Collective Behavior", en A. Mcclung (Ed.). Principles of Sociology. Nueva York, Barnes \& Noble, pp. 67-121.

CAMINAL, Miquel (2005), Manual de Ciencia Política. Madrid, Tecnos.

CASADO, Cristina; COLOMO, Ricardo (2006), "Un breve recorrido por la concepción de las emociones en la filosofía occidental". A parte Rei. Revista de filosofía. Madrid, v. 47, pp. 1-9[Consult. 05-052020]. Disponible en http://serbal.pntic.mec.es/ cmunoz11/casado47.pdf

CASTEL, Robert; CASTEL, François; LOVELL, Anne (1982), The Psychiatric Society. Nueva York, Columbia University Press.

CASTELLS, Manuel (1977a), "Les conditions sociales d'emergence des mouvements sociaux urbains". International Journal of Urban and Regional Research. Hoboken, v. 1, pp. 45-60 [Consult. 01-062020]. Disponible em https://onlinelibrary.wiley.com/doi/epdf/10.1111/j.1468-2427.1977.tb00698.x

CASTELLS, Manuel (1977b), "Marginalite urbaine et mouvements sociaux au Mexique: le mouvement des posesionarios dans la ville de Monterrey". International Journal of Urban and Regional Research. Hoboken, v. 2, pp. 145-50 [Consult. 11-04-2020]. Disponible en https://onlinelibrary.wiley.com/doi/epdf/10.1111/j.1468-2427.1977.tb00703.x

CASTELLS, Manuel (1996), La era de la información: economía, sociedad y cultura I: La Sociedad Red. Madrid, Alianza.

CASTELLS, Manuel (2006), La Sociedad Red: una visión global. Madrid, Alianza.

CASTELLS, Manuel (2012), Redes de indignación y esperanza. Madrid, Alianza.

CASTELLS, Manuel (2013), La era de la información: El poder de la identidad. Madrid, Alianza.

DAHL, Robert (1961), "The behavioral approach in political science: epitaph for a monument to a successful protest". American Political Science Review. Cambridge, v. 55, 4, pp. 763-772 [Consult. 10-03-2020]. Disponible en https://www.jstor.org/stable/1952525?seq=1\#metadata_info_tab_contents

DAMASIO, Antonio R. (2013), En busca de Spinoza. Neurobiología de la emoción y los sentimientos. Barcelona, Destino.

DE LA GARZA, Rafael (2011), "Las teorías de los movimientos sociales y el enfoque multidimensional”. Estudios políticos. México, v. 22, pp. 107-138 [Consult. 09-02-2020]. Disponible en https://www.jstor.org/stable/1952525?seq=1\#metadata_info_tab_contents 
DE SAUSSURE, Ferdinand (1987), Curso de lingüística general. Madrid, Alianza.

DE SOUSA, Boaventura (2001), "Los nuevos movimientos sociales". Observatorio Social de América Latina. Buenos Aires, v. 5, pp. 177-188 [Consult. 07-03-2020]. Disponible en http://www.boaventuradesousasantos.pt/media/pdfs/Los_nuevos_movimientos_sociales_OSAL2001.P $\mathrm{DF}$

DELLA PORTA, Donatella; DIANI, Mario (2011), Los Movimientos Sociales. Madrid, Centro de Investigaciones Sociológicas.

DESCARTES, René (1989) [1628], Reglas para la dirección del espíritu. Madrid, Alianza.

DIANI, Mario (1992), “The Concept of Social Movement”. Sociological review. Londres, v. 40, 1, pp. 125.

DIZ, Isabel, LOIS, Marta; NOVO, Amparo (2004), Elementos Fundamentales de Ciencia Política. Santiago de Compostela, Tórculo.

EASTON, David (1953), The Political System, Nueva York, Knofp.

EISINGER, Peter K. (1973), "The conditions of protest behavior in American cities". American political science review. Cambridge, v.67, 1, pp. 11-28 [Consult. 18-04-2020]. Disponible en https://www.jstor.org/stable/1958525?seq=1\#metadata_info_tab_contents

EKMAN, Paul (1999), "Basic Emotions”, en T. Dalgleish, M. Power (Eds.). Handbook of Cognition and Emotion. Sussex, Wiley and Sons, pp. 45-60.

EKMAN, Paul (2017), El rostro de las emociones. Qué nos revelan las expresiones faciales. Barcelona, RBA.

EKMAN, Paul; LEVENSON, Robert W.; FRIESEN, Wallace V. (1983), “Autonomic nervous system activity distinguishes among emotions”. Science. Washington, v. 221, 4616, pp. 1208-1210 [Consult. 11-05-2020].

Disponible

em https://science.sciencemag.org/content/221/4616/1208\#: :text=The\%20autonomic\%20activity\%20pro duced\%20distinguished,of\%20autonomic\%20differentiation\%20in\%20emotion

ELSTER, Jon (2002), Alquimias de la mente. La racionalidad y las emociones. Barcelona, El Roure.

ELSTER, Jon (2010), La explicación del comportamiento social. Barcelona, Gedisa.

ENCISO, Giazú; LARA, Alí (2013). "El giro afectivo". Athenea Digital: revista de pensamiento e investigación social. Cerdanyola del Vallès, v.13, 3, pp. 101-120 [Consult. 15-07-2020]. Disponible enhttps://atheneadigital.net/article/view/v13-n3-lara-enciso.

ENCISO, Giazú; LARA, Alí (2014), "Emociones y ciencias sociales en el S. XX: la precuela del giro afectivo". Athenea digital: revista de pensamiento e investigación social. Cerdanyola del Vallès, v. 14, $1, \quad$ pp. 263- 288 [Consult. 08-08-2020]. Disponible en https://www.researchgate.net/publication/269749853_Emociones_y_ciencias_sociales_en_el_s_XX_L a_precuela_del_giro_afectivo

EYERMAN, Ron; JAMISON, Andrew (1991), Social movements. A Cognitive approach. Cambridge, Polity Press.

FERRATER, José (1988), Diccionario de Filosofía (V. II). Barcelona, Ariel, pp. 915-917. 
FREEDEN, Michael (2013), The political theory of political thinking: The anatomy of a practice. Oxford, Oxford University Press.

FUNES, M.J.; MONFERRER, J. (2003), "Perspectivas teóricas y aproximaciones metodológicas al estudio de la participación", en M. J. Funes, R. Adell (Eds.), Movimientos sociales: cambio social y participación. Madrid, Universidad Nacional de Educación a Distancia, pp. 21-58.

GABRIEL, Markus (2019), El sentido del pensamiento. Barcelona, Ediciones de Pasado y Presente.

GAMSON, W.A.; MEYER, D.S. (1996), "Framing Political Opportunity", en D. McAdam, J. D. McCarthy,M. N. Zald (Eds.) Comparative Perspectives on Social Movements. Cambridge, Cambridge University Press, pp. 275-290.

GOODWIN, Jeff; JASPER, James M.; POLLETTA, Francesca (2000), "Return of the Repressed: The Fall and Rise of Emotions in Social Movement Theory". Mobilization: An International Journal. San Diego, v.5, pp. 65-82 [Consult. 17-07-2020]. Disponible en http://www.socsci.uci.edu/ polletta/Articles\%20and\%20Book\%20Chapters_files/Return_of_represse d.pdf

GOODWIN, J.; JASPER, J.M. (2004), "Caught in a Winding, Snarling Vine: The Structural Bias of Political Process Theory”, en J. Goodwin, J. M. Jasper (Eds.). Rethinking Social Movements. Lanham, Rowman y Littlefield, pp. 27-136.

GUIBERNAU, Montserrat (2017), Identidad, pertenencia, solidaridad y libertad en las sociedades modernas. Madrid, Trotta.

HUMPRHREY, Nicholas (2012), “Un yo que vale la pena”, en J. Brockman (Ed.). Mente. Barcelona, 235 Crítica, pp. 121-135.

INGLEHART, Ronald (1971), "The silent revolution in post-industrial societies". American Political Science Review. Cambridge, v.65, pp. 991-1017 [Consult. 13-07-2020]. Disponible en https://www.jstor.org/stable/1953494?seq=1\#metadata_info_tab_contents

INGLEHART, Ronald (1998), Modernización y posmodernización: el cambio cultural, económico y político en 43 sociedades. Madrid, Centro de Investigaciones Sociológicas.

JACK, Rachael E.; GARROD, Oliver G.B.; SCHYNS, Philippe G. (2014), "Dynamic Facial Exppresion of Emotion Transmit an Evolving Hierarchy of Signals over Time". Current Biology. Amsterdam, v.24, pp. 187-192 [Consult. 11-07-2020]. Disponible en https://doi.org/10.1016/j.cub.2013.11.064

JASPER, James M. (1997), The Art of Moral Protest: Culture, Biography, and Creativity in Social Movements. Chicago, Chicago University Press.

JASPER, James M. (2012a), "Las emociones y los movimientos sociales: veinte años de teoría e investigación". Revista Latinoamericana de Estudios sobre Cuerpos, Emociones y Sociedad. Córdoba, v.4, 10, pp. 46-66 [Consult. 11-06-2020]. Disponible en https://www.redalyc.org/articulo.oa?id=273224904005

JASPER, James M. (2012b), “¿De la estructura de la acción? La teoría de los movimientos sociales depués de los grandes paradigmas". Sociológica, v.27, 75, pp. 7-48 [Consult. 11-7-2020]. Disponible en http://www.scielo.org.mx/scielo.php?script=sci_arttext\&pid=S0187-01732012000100001

JASPER, James M. (2012c), "From Macro Opportunities to Micro Mechanisms", en J. Goodwin, J. M. Jasper (Eds.). Contention in Context. Stanford, Stanford University Press, pp. 2-29. 
KRIESI, Hanspeter (1989), "New social movements and the New Class in the Netherlands". American Journal of Sociology. Chicago, v.95, 5, pp. 1078-1116 [Consult. 03-07-2020]. Disponible en https://www.jstor.org/stable/2780467?seq=1\#metadata_info_tab_contents

LABORA, Juan J. (2018), A evolución da percepción social dos trastornos da conducta alimentaria dos profesionais da saúde en Galicia. Santiago de Compostela, Universidade de Santiago de Compostela

LASWELL, Harold; KAPLAN, Abraham (1950), Power and Society. New Haven, Yale University Press.

LAZARSFELD, Paul F.; BERELSON, Bernard; GAUDET, Hazel (1948), The people's choice. Nueva York, Columbia University Press.

LAZARUS Richard S. (1993), "From psychological stress to the emotions: a history of changing outlooks". Annual Review of Psychology. Palo Alto, 44, pp. 1-21 [Consult. 17-06-2020]. Disponible en https://www.annualreviews.org/doi/abs/10.1146/annurev.ps.44.020193.000245

LEDOUX, J. (2012), "Recuerdos paralelos: devolviendo las emociones al cerebro". En BROCKMAN, John (Ed.). Mente. Madrid, Crítica, pp. 35-53.

LIPSKY, Michael (1965), Protest and city politics. Chicago, Rand McNally.

MÁIZ, Ramón (2010), "La hazaña de la razón: la exclusión fundacional de las emociones en la teoría política moderna". Revista de estudios políticos. Santiago de Compostela, v.149, pp. 11-45 [Consult. 15-11-2020]. Disponible en https://dialnet.unirioja.es/servlet/articulo?codigo=3301555

MANEIRO, Elba (2017), "Neurociencia y emociones: nuevas posibilidades en el estudio del comportamiento político". RIPS: Revista de Investigaciones Políticas y Sociológicas. Santiago de Compostela, v.16, 1, pp. 169- 187 [Consult. 10-07-2020]. Disponible en https://doi.org/10.15304/rips.16.1.4018

MARCUS, G. (2003), "The psychology of emotion and politics". En SEARS, David O.; HUDDY, Leonie; JERVIS, Robert (Eds.), Oxford handbook of political psychology. Oxford University Press. pp. 182-221.

MARCUS, George; NEUMAN, Rusell; CRIGLER, Ann N.; MACKUEN, Michael (2007), The Affect Effect: dynamics of emotion in political thinking and behaviour. Chicago, University of Chicago Press.

MARINA, José A. (2005), El laberinto sentimental. Barcelona. Anagrama.

MARINA, José A.; LÓPEZ, Marisa (1999), Diccionario de los sentimientos. Barcelona, Círculo de lectores.

MATURANA, Humberto A. (1997), Emociones y lenguaje en educación y política. Santiago de Chile, Dolmen.

MAZZOLENI, Gianpietro (2010), La comunicación política. Madrid, Alianza.

MCADAM, Doug. (1982), Political Process and the Development of Black Insurgency, 1930-1970. Chicago, Chicago University Press.

MCCARTHY, John; ZALD, Mayer N. (1977), "Resource Mobilization and Social Movements: A Partial Theory". American Journal of Sociology. Chicago, v.82, pp. 1212-1241 [Consult. 03-07-2020]. Disponible en https://www.jstor.org/stable/2777934?seq=1\#metadata_info_tab_contents 
MELUCCI, Alberto (1980), "The new social movements: A theoretical approach". Information (International Social Science Council). París, v. 19, 2, pp. 199-226 [Consult. 11-10-2020]. Disponible en https://journals.sagepub.com/doi/10.1177/053901848001900201

MELUCCI, Alberto (1982), Sistema politico, partiti e movimenti sociali. Milán, Feltrinelli.

MELUCCI, Alberto (1989), Nomads of the present. Social movements and individual nerds in contemporary society. Londres, Hutchinson.

MELUCCI, Alberto (1995), "The process of collective identity". Social movements and culture. Minneapolis, v.4, pp. 41- 63 [Consult. 17-03-2020]. Disponible en https://pages.ucsd.edu/ jhaydu/melucci-identity.pdf

MELUCCI, Alberto (1996), Challenging codes: Collective action in the information age. Cambridge, Cambridge University Press.

MELUCCI, Alberto (2001), Vivencia y convivencia: teoría social para una era de la información. Madrid, Trotta.

MONFERRER, J.M. (2006), "Tipología de los movimientos sociales en relación al cambio". En MARÍN, Antonio L. (Coord.). Estructura social. La realidad de las sociedades avanzadas. Madrid, Prentice Hall, pp. 379-412.

MONTERO, José R.; TORCAL, Mariano (1995). "Cambio cultural, conflictos políticos y política en España". Revista de estudios políticos, n. 89, pp. 9-34 [Consult. 15-09-2020]. Disponible en https://dialnet.unirioja.es/servlet/articulo?codigo=27348

MORIN, Edgar (2014), El Método. La humanidad de la humanidad. La identidad humana. Madrid, Cátedra.

OBERSCHALL, Anthony (1995), Social movements: ideologies, interest, and identities. Nuevo Brunswick, Transaction Publishers.

OFFE, Claus (1992), Partidos políticos y nuevos movimientos sociales. Madrid, Sistema.

OLSON, Marcur (1965), The Logic of Collective Action. Harvard, Harvard University Press.

OPP, Karl-Dieter (2009), Theories of political protest and social movements. Londres, Routledge.

PERROW, Charles; JENKINS, Craig (1977), "Insurgency of the powerless: Farm worker movements (1946-1972)". American sociological review. Washington, v.42, 2, pp. 249-268 [Consult. 16-102020]. Disponible en https://www.jstor.org/stable/2094604?seq=1\#metadata_info_tab_contents

PIVEN, F.; CLOWARD, R. (1992), "Normalizing Collective Protest". En MORRIS, Aldon D.; MUELLER, Carol (Eds.), Frontiers of Social Movement Theory. New Haven, Yale University Press. pp. 301-325.

PLUTCHIK, Robert (2001), "The nature of emotions: Human emotions have deep evolutionary roots, a fact that may explain their complexity and provide tools for clinical practice". American scientist. v.89, 4, pp. 344-350 [Consult. 12-011-2020]. Disponible en https://www.jstor.org/stable/27857503?seq=1

PLUTCHIK, Robert (2003), Emotions and life: Perspectives from psychology, biology, and evolution. Washington, American Psychological Association. 
SÁNCHEZ, María J. (2016), "El giro emotivo del espacio público. Corazonadas y subjetividades". En PEÑAMARÍN, Cristina (Coord.), Emociones en la nueva esfera pública. Federación Latinoamericana de Semiótica, pp. 147-160.

SCULL, Andrew (1989), Social Order/Mental Disorder. Anglo-American Psychiatry in Historical perspective. Los Angeles, University of California Press.

SQUIRE, Corinne (2001), "The public life of emotions". International Journal of Critical Psychology. v.1, pp. 27-38 [Consult. 19-02-2020]. Disponible en https://www.researchgate.net/publication/47528921_The_Public_Life_of_Emotions

STEARNS, Peter Z; STEARNS, Carol Z. (1985); "Emotionology: Clarifying the history of emotions and emotional standards". The American Historical Review. Washington, v. 90, 4, pp. 813-836.

SUTIL, Carlos (2013). “QQué es una emoción? Teoría relacional de las emociones”. Clínica e Investigación relacional. v. 7, 2, pp. 348-372 [Consult. 15-04-2020]. Disponible en https://www.psicoterapiarelacional.es/Portals/0/eJournalCeIR/V7N2_2013/10-Rodriguez-Sutil_Quees-una-emocion_CeIR_V7N2.pdf

SZTOMPKA, Piotr (1995), Sociología del cambio social. Madrid, Alianza.

TARROW, S.G. (2002), "Ciclos de acción colectiva: entre los momentos de locura y el repertorio de contestación". En Traugott, Mark (Comp.), Protesta social. Repertorios y ciclos de la acción colectiva. Barcelona, Hacer, pp. 99-130.

TARROW, Sidney G. (2012), El poder en movimiento: Los movimientos sociales, la acción colectiva y la política. Madrid, Alianza.

TAYLOR, V.; VAN DYKE, N. (2004), "«Get up, stand up»: Tactical repertoires of social movements", en D. A. Snow, S. A. SoulE, H. Kriesi (Eds.), The Blackwell companion to social movements. Oxford, Blackwell, pp. 262-293.

TILLY, Charles (1978), From Mobilization to Revolution. Reading, Mass.

TORREBlanCA, I. (2006), "La Ciencia Política empírica II: enfoques de investigación”, en M. J. Sodaro (Ed.). Política y Ciencia Política. Madrid, McGraw-Hill, pp. 57-76.

TORRES, Manuel (2012), "Imaginarios sociales de la enfermedad mental". Revista de investigaciones políticas y sociológicas. Santiago de Compostela, v.11, 2, pp. 101-113 [Consult. 13-07-2020]. Disponible en https://revistas.usc.gal/index.php/rips/article/view/378

TOURAINE, Alain (1994), Crítica de la modernidad. México, Fondo de Cultura Económica.

TOURAINE, Alain (1995), Producción de la sociedad. México, Universidad Nacional Autónoma de México.

ULLÁN, Francisco J. (2016), Teorías sociológicas de los movimientos sociales. Madrid, Catarata.

USSHER, Jane (1991), Women's Madness. Nueva York, Harvester Wheatsheaf.

ZUBIRI, Xavier (1982), Inteligencia y logos. Madrid, Alianza/Sociedad de Estudios y Publicaciones.

ZUBIRI, Xavier (1983), Inteligencia y razón. Madrid, Alianza/Sociedad de Estudios y Publicaciones.

ZUBIRI, Xavier (1991), Inteligencia y realidad. Madrid, Alianza/Fundación Xavier Zubiri. 


\begin{abstract}
This paper analyzes the primary emotions and emotional components, which influence social behavior at the individual and collective level and how they affect the study of New Social Movements (NSM). As opposed to the predominant rationalism in analysing these types of social action, the analysis of the role of emotions was chosen. To achieve these objectives, a bibliographic review focuses on the debate between the predominant rationalism in the study of traditional social movements and the approaches that place the role of emotions in the new social movements at the centre of the research. Finally, the new social movements are characterized as forms of action, with some characteristics that differentiate them from traditional movements, mainly because they are identity movements.
\end{abstract}

Keywords: emotions, new social movements, collective action; social identity.

\title{
Resumo
}

Este artigo analisa as principais emoções e componentes emocionais que influenciam o comportamento social a nível individual e coletivo e como afetam o estudo dos Novos Movimentos Sociais (NMS). Contra o racionalismo predominante na análise desses tipos de ação social, optou-se pela análise do papel das emoções. Para atingir esses objetivos, foi realizada uma revisão bibliográfica com foco no debate entre o racionalismo predominante na análise dos movimentos sociais tradicionais e as abordagens que colocam o papel das emoções nos novos movimentos sociais no centro da pesquisa. Por fim, compreendese que os novos movimentos sociais se caracterizam como formas de ação, com algumas características que os diferenciam dos movimentos tradicionais, principalmente pelo fato de serem movimentos identitários.

Palavras-chave: emoções; novos movimentos sociais; ação coletiva; identidade social. 